\title{
POST-OPERATIVE COMPLICATIONS IN TRAM FLAP BREAST RECONSTRUCTION AFTER MASTECTOMY
}

doi: 10.2478/rojost-2018-0058

\author{
S.V. Parasca, B.F. Iosif, A. Costeschi, O. Popescu, M. Roznovanu
}

Emergency Hospital for Plastic Reconstructive Surgery and Burncare, Bucharest, Romania

Introduction. Breast cancer is the main cause of mortality for neoplastic diseases in women worldwide. Since the incidence is increasing every year, the need for reconstructive techniques increases and autologous tissue reconstruction procedures are also gaining ground over the more popular expander/ implant techniques.

A hypertensive and obese patient is admitted by SCUCPRA's Chronic Diseases Service, with a postmastectomy scar on the left breast, with normal aspect. A TRAM Flap is performed through an oblique tunnel for transposing the tissue over the defect. The abdominal defect was covered with polypropylene mesh. Three days post-operatory, the patient presented an umbilical necrotic scar of $6 / 3 \mathrm{~cm}$ and a $4 / 3 \mathrm{~cm}$ necrotic scar in the medial zone of the TRAM Flap.

Materials and method. Four days post-operatory, the patient presented a $10 / 5 \mathrm{~cm}$ brown-black colored dehiscent umbilical scar and 2 dehiscent areas of $4 / 1 \mathrm{~cm}$ and $5 / 1 \mathrm{~cm}$ inferior and superior, on the TRAM Flap. Excisional debridement of the ulceration was performed, and the abdominal defect was covered with a split-thickness skin graft. The TRAM Flap was also debrided and immediate suture was performed.

Conclusions and results. Necrosis is an important complication that can occur both at the abdominal level, as well as on the TRAM Flap, after a breast reconstruction, affecting both the general state of the patient and the long-term results of the reconstructive method.

Keywords: necrosis, TRAM Flap 